\title{
Transport, Aharonov-Bohm, and Topological Effects in Graphene Molecular Junctions and Graphene Nanorings
}

\author{
Constantine Yannouleas, Igor Romanovsky, and Uzi Landman* \\ ${ }^{\dagger}$ School of Physics, Georgia Institute of Technology, Atlanta, Georgia 30332-0430, United States
}

\begin{abstract}
The unique ultrarelativistic, massless, nature of electron states in two-dimensional extended graphene sheets, brought about by the honeycomb lattice arrangement of carbon atoms in two dimensions, provides ingress to explorations of fundamental physical phenomena in graphene nanostructures. Here, we explore the emergence of new behavior of electrons in atomically precise segmented graphene nanoribbons (GNRs) and graphene rings with the use of tight-binding calculations, nonequilibrium Green's function transport theory, and a newly developed Dirac continuum model that absorbs the valence-to-conductance energy gaps as position-dependent masses, including topological-in-origin mass barriers at the contacts between

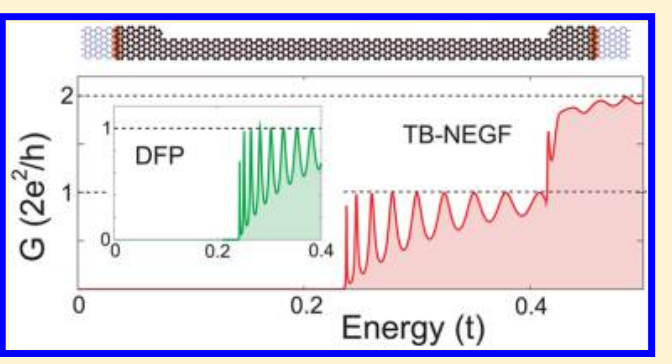
segments. Through transport investigations in variable-width segmented GNRs with armchair, zigzag, and mixed edge terminations, we uncover development of new Fabry-Pérot-like interference patterns in segmented GNRs, a crossover from the ultrarelativistic massless regime, characteristic of extended graphene systems, to a massive relativistic behavior in narrow armchair GNRs, and the emergence of nonrelativistic behavior in zigzag-terminated GNRs. Evaluation of the electronic states in a polygonal graphene nanoring under the influence of an applied magnetic field in the Aharonov-Bohm regime and their analysis with the use of a relativistic quantum field theoretical model unveils development of a topological-in-origin zero-energy soliton state and charge fractionization. These results provide a unifying framework for analysis of electronic states, coherent transport phenomena, and the interpretation of forthcoming experiments in segmented GNRs and polygonal rings.
\end{abstract}

\section{INTRODUCTION}

In the last 3 decades, transport through molecular junctions ${ }^{1-6}$ has attracted much attention because of fundamental aspects of the processes involved, as well as of potential practical prospects. In particular, studies in this direction have intensified since the discovery of new forms of carbon allotropes, starting with the fullerenes ${ }^{7}$ and carbon nanotubes ${ }^{8}$ (CNTs) in the 1980 s and 1990s, respectively, and the isolation of graphene ${ }^{9}$ in 2004. The above carbon allotropes differ in shape (curvature), topology, and dimensionality, with the fullerenes being zerodimensional (OD) with spherical or prolate shape, the CNTs being one-dimensional (1D) cylinders, and graphene being a two-dimensional (2D) plane (or 1D planar ribbons ${ }^{10-13}$ ). In the fullerenes, the carbon atom network is made of (nonadjacent) hexagons and pentagons, whereas the CNTs and graphene are entirely hexagonal lattices (curved in CNTs) described in terms of a unit cell with a two-atom basis with the two carbon atoms occupying two sublattices ( $A$ and $B$, also mapped into the up and down pseudospin states $\left.{ }^{14}\right)$. In the absence of defects, in-plane $(\sigma)$ bonding occurs through $\mathrm{sp}^{2}$ hybrid orbitals, and out-of-plane bonding $(\pi)$ involves the $\mathrm{p}_{z}$ orbital; in the following, only the physics of $\pi$-states is considered.

The hexagonal network topology of graphene gives rise to relativistic behavior of the low-energy excitations, which is captured by the ultrarelativistic massless Dirac-Weyl (DW) equation, with the Fermi velocity of graphene $\left(v_{\mathrm{F}}=c / 300\right)$ replacing the velocity of light. ${ }^{14}$ Among the many manifestations of the relativistic behavior in graphene is the Klein paradox, that is, "... unimpeded penetration of relativistic particles through high and wide potential barriers-is one of the most exotic and counterintuitive consequences of quantum electrodynamics." 15 The surprising relativistic behavior in graphene has indeed been recognized in the 2010 Nobel award in physics to A. Geim and K. Novosolev.

Another carbon-based system that was the subject of an earlier (2000) chemistry Nobel award (to A. J. Heeger, A. G. MacDiarmid, and H. Shirakawa) is conductive polymers, with polyacetelyne (PAC) being a representative example. ${ }^{16} \mathrm{PAC}$ is a $1 \mathrm{D}$ chain of carbon atoms forming a conjugated polymer with $\mathrm{sp}^{2} \mathrm{p}_{z}$ hybridization that leads to one unpaired electron per carbon atom (half-filled $\pi$-band), as in graphene. Linearizing the spectrum of this $1 \mathrm{D}$ equally spaced carbon chain at the Fermi level (that is, at the Dirac points, i.e., the zero-energy points of the energy versus momentum dispersion relation) results in a $1 \mathrm{D}$ massless Dirac equation description of the lowenergy excitations of the system. ${ }^{17,18}$

However, the equally spaced 1D system is unstable, and consequently, it distorts (structurally) spontaneously (Peierls distortion ${ }^{19}$ ), yielding a modulation (alternation) of the spacing between successive sites that results in dimerization of

Special Issue: Current Trends in Clusters and Nanoparticles Conference

Received: November 30, 2014

Revised: January 7, 2015

Published: January 28, 2015 
successive atoms along the chain and the opening of a gap in the electronic spectrum. This dimerization can occur in two energetically degenerate, but spatially distinct, patterns, termed as equivalent domain structures. Either of these domains is a realization of the $1 \mathrm{D}$ Dirac equation with a constant mass term, $\mathcal{M}$. Disruption of the dimerization pattern (e.g., by transforming from one domain to another along the chain) creates a domain wall that can be described in the 1D Dirac formulation through the use of a position-dependent mass term of alternating sign (with the spatial mass profile connecting $+\mathcal{M}$ with $-\mathcal{M})$. The solution of this generalized 1D Dirac equation is a soliton characterized by having zero energy and by being localized at the domain wall. In this paper, the Dirac equation with position-dependent mass will be used in investigations of the electronic structure, transport, and magnetic-field-induced phenomena (Aharonov-Bohm, AB) in graphene nanostructures, such as nanoribbon junctions and rings. ${ }^{20-22}$

From the above, we conclude that in the two extreme size domains, that is, a $2 \mathrm{D}$ infinite graphene sheet and a $1 \mathrm{D}$ carbon chain (PAC), the systems exhibit behavior that is relativistic in nature. In an attempt to bridge between these two size domains, we briefly discuss in the following systems of successively larger width, starting from the polyacene (a quasi-1D chain of fused benzene rings) that may be regarded as the narrowest graphene nanoribbon (GNR) with zigzag edge terminations.

Polyacene was investigated ${ }^{23}$ first in 1983. It was found that, as in graphene and PAC (without distortion), the valence and conduction bands of undistorted polyacene touch at the edge of the Brillouin zone. However, unlike 2D graphene and PAC, the dispersion relation about the touching point is quadratic, conferring a nonrelativistic (Schrödinger equation) character. We show in this paper that this surprising finding persists for sufficiently narrow GNRs with zigzag termination (zGNRs). However, narrow armchair-terminated GNRs (aGNRs) are found here to maintain relativistic behavior, with metallic ones being massless and semiconducting ones being massive (both classes obeying the Einstein energy relation).

It this paper, we discuss mainly manifestations of relativistic and/or nonrelativistic quantum behavior explored through theoretical considerations of transport measurements in segmented GNRs of variable width and spectral and topological effects in graphene nanorings in the presence of magnetic fields.

Transport through narrow graphene channels, particularly bottom-up fabricated and atomically precise GNRs, ${ }^{24-31}$ is expected to offer ingress to unique behavior of Dirac electrons in graphene nanostructures. In particular, the wave nature of elementary particles (e.g., electrons and photons) is commonly manifested and demonstrated in transport processes. Because of an exceptionally high electron mobility and a long mean-free path, it has been anticipated that graphene ${ }^{14}$ devices hold promise for the realization, measurement, and possible utilization of fundamental aspects of coherent and ballistic transport behavior, which to date have been observed, with varying degrees of success, mainly at semiconductor interfaces, ${ }^{32,33}$ quantum point contacts, ${ }^{34}$ metallic wires, ${ }^{35}$ and carbon nanotubes. ${ }^{36}$

Another manifestation of coherent ballistic transport are interference phenomena, reflecting the wave nature of the transporting physical object and associated most often with optical (electromagnetic waves, photons) systems or with analogies to such systems (that is, the behavior of massless particles, as in graphene sheets). Measurements of interference patterns are commonly made with the use of interferometers, most familiar among them the multipass optical Fabry-Pérot (OFP) interferometer. ${ }^{37,38}$ The advent of $2 \mathrm{D}$ forms of carbon allotropes has motivated the study of optical-like interference phenomena associated with relativistic massless electrons, as in the case of metallic carbon nanotubes ${ }^{36}$ and graphene $2 \mathrm{D} p-n$ junctions. ${ }^{39}$ (We note that the hallmark of the OFP is that the energy separation between successive maxima of the interference pattern varies as the inverse of the cavity length $L$.)

For GNRs with segments of different widths, our investigations reveal diverse Fabry-Pérot (FP) transport modes beyond the OFP case, with conductance quantization steps $\left(n G_{0}, n=1,2,3, \ldots\right.$, with $\left.G_{0}=2 e^{2} / h\right)$ found only for uniform GNRs. In particular, three distinct categories of FP interference patterns are identified:

(1) FP-A: An optical FP pattern corresponding to massless graphene electrons exhibiting equal spacing between neighboring peaks. This pattern is associated with metallic armchair nanoribbon central segments. This category is subdivided further to FP-A1 and FP-A2, depending on whether a valenceto-conduction gap is absent ( $F P-A 1$, associated with metallic armchair leads) or present (FP-A2, corresponding to semiconducting armchair leads).

(2) FP-B: A massive relativistic FP pattern exhibiting a shift in the conduction onset due to the valence-to-conduction gap and unequal peak spacings. This pattern is associated with semiconducting armchair nanoribbon central segments, irrespective of whether the leads are metallic armchair, semiconducting armchair, or zigzag.

(3) FP-C: A massive nonrelativistic FP pattern with $1 / L^{2}$ peak spacings but with a vanishing valence-to-conduction gap, $L$ being the length of the central segment. This pattern is the one expected for usual semiconductors described by the (nonrelativistic) Schrödinger equation, and it is associated with zigzag nanoribbon central segments, irrespective of whether zigzag or metallic armchair leads are used.

We report in this paper on the unique apects of transport through segmented GNRs obtained from tight-binding (TB) nonequilibrium Green's function ${ }^{40,41}$ (TB-NEGF) calculations in conjunction with an analysis based on a $1 \mathrm{D}$ relativistic Dirac continuum model. This continuum model goes beyond the physics of the massless DW electron, familiar from 2D honeycomb carbon sheets, ${ }^{14}$ and it is referred to by us as the Dirac-Fabry-Pérot (DFP) theory (see below for the choice of name). In particular, it is shown that the valence-to-conduction energy gap in aGNR segments, as well as the barriers at the interfaces between nanoribbon segments, can be incorporated in an effective position-dependent mass term in the Dirac Hamiltonian; the transport solutions associated with this Hamiltonian exhibit conductance patterns comparable to those obtained from the microscopic NEGF calculations. For zGNR segments, the valence-to-conduction energy gap vanishes, and the mass term is consonant with excitations corresponding to massive nonrelativistic Schrödinger-type carriers. The faithful reproduction of these unique TB-NEGF conductance patterns by the DFP theory, including mixed armchair-zigzag configurations (where the carriers transit from a relativistic to a nonrelativistic regime), provides a unifying framework for analysis of coherent transport phenomena and for interpretation of experiments targeting fundamental understanding of transport in GNRs and the future development of graphene nanoelectronics. 
To demonstrate the aforementioned soliton formation due to structural topological effects (discussed by us above in the context of polyacetylene), we explore with numerical TB calculations and a Dirac-Kronig-Penney (DKP) approach soliton formation and charge fractionization in graphene rhombic rings; this DKP approach is based on a generalized Dirac equation with alternating-sign position-dependent masses.

Before leaving the Introduction, we mention that, due to their importance as fundamental phenomena, AB-type effects in GNR systems have attracted (in addition to refs 20-22) considerable theoretical attention. ${ }^{4,42-47}$ These latter theoretical papers, however, based their analysis exclusively on TB and/ or DFT calculations, ${ }^{4,42,44,46,47}$ or they used in addition a $2 \mathrm{D}$ Dirac equation with infinite-mass boundary conditions. ${ }^{43,45}$ Transcending the level of current understanding that explores direct similaritiess with the $\mathrm{AB}$ physics in semiconductor and metallic mesoscopic rings, our work here analyzes the TB results in conjunction with a continuum $1 \mathrm{D}$ generalized Dirac equation (that incorporates a position-dependent mass term), and thus, it enables investigations of until-now unexplored topological aspects and relativistic quantum field analogies of the $A B$ effect in graphene nanosystems.

Furthermore, we note that oscillations in the conductance of GNRs in the presence of magnetic barriers were found in a theoretical study ${ }^{48}$ (using exclusively a TB-NEGF approach), as well as in an experimental investigation of high-quality bilayer nanoribbons, ${ }^{49}$ and they were attributed to FP-type interference. In the absence of a continuum Dirac analysis, the precise relation of such oscillatory patterns to our FP theory (based on the incorporation of a position-dependent mass term in the Dirac equation at zero magnetic field) warrants further investigation.

Finally, gap engineering in graphene ribbons under strong external fields was studied in ref 50 . We stress again that one of the main results in this paper is the appearance of "forbidden" solitonic states inside of the energy gap in the context of the low-magnetic-field $A B$ spectra of graphene nanorings; see the part titled "AB Spectra of Rhombic Graphene Rings" in the Results section.

\section{METHODS SECTION}

DFP Model. The energy of a Fermion (with 1D momentum $\left.\mathrm{p}_{x}\right)$ is given by the Einstein relativistic relation $E=\left[\left(\mathrm{p}_{x} \nu_{\mathrm{F}}\right)^{2}+\right.$ $\left.\left(\mathcal{M} \nu_{\mathrm{F}}^{2}\right)^{2}\right]^{1 / 2}$, where $\mathcal{M}$ is the rest mass and $\nu_{\mathrm{F}}$ is the Fermi velocity of graphene. In a gapped uniform GNR, the mass parameter is related to the particle-hole energy gap, $\Delta$, as $\mathcal{M}=$ $\Delta /\left(2 \nu_{\mathrm{F}}^{2}\right)$. Following the relativisitic quantum field Lagrangian formalism, the mass $\mathcal{M}$ is replaced by a position-dependent scalar Higgs field $\phi(x) \equiv m(x) \nu_{\mathrm{F}}^{2}$, to which the relativistic Fermionic field $\Psi(x)$ couples through the Yukawa Lagrangian ${ }^{21}$ $\mathcal{L}_{\mathrm{Y}}=-\phi \Psi^{\dagger} \beta \Psi$ ( $\beta$ being a Pauli matrix). For $\phi(x) \equiv \phi_{0}$ (constant) $\mathcal{M} \nu_{\mathrm{F}}^{2}=\phi_{0}$, and the massive Fermion Dirac theory is recovered. The Dirac equation is generalized as (here we do not consider applied electric or magnetic fields)

$$
[E-V(x)] \Psi+\mathrm{i} \hbar \nu_{\mathrm{F}} \alpha \frac{\partial \Psi}{\partial x}-\beta \phi(x) \Psi=0
$$

In one dimension, the Fermion field is a two-component spinor $\Psi=\left(\psi_{\mathrm{u}} \psi_{1}\right)^{T} ; \mathrm{u}$ and 1 stand, respectively, for the upper and lower components, and $\alpha$ and $\beta$ can be any two of the three Pauli matrices. Note that the Higgs field enters in the last term of eq 1. $V(x)$ in the first term is the usual electrostatic potential, which is inoperative due to the Klein phenomenon and thus is set to zero for the case of the armchair nanoribbons (where the excitations are relativistic). The generalized Dirac eq 1 is used in the construction of the transfer matrices of the DFP model described below.

The building block of the DFP model is a $2 \times 2$ wave function matrix $\Omega$ formed by the components of two independent spinor solutions (at a point $x$ ) of the onedimensional, first-order generalized Dirac equation [see eq 3 in the main paper]. $\Omega$ plays ${ }^{51}$ the role of the Wronskian matrix $\mathbf{W}$ used in the second-order nonrelativistic Kronig-Penney model. Following ref 51 and generalizing to $N$ regions, we use the simple form of $\Omega$ in the Dirac representation $\left(\alpha=\sigma_{1}, \beta=\sigma_{3}\right)$, namely

$$
\Omega_{K}(x)=\left(\begin{array}{cc}
\mathrm{e}^{\mathrm{i} K x} & \mathrm{e}^{-\mathrm{i} K x} \\
\Lambda \mathrm{e}^{\mathrm{i} K x} & -\Lambda \mathrm{e}^{-\mathrm{i} K x}
\end{array}\right)
$$

where

$$
K^{2}=\frac{(E-V)^{2}-m^{2} \nu_{\mathrm{F}}^{4}}{\hbar^{2} \nu_{\mathrm{F}}^{2}} \quad \Lambda=\frac{\hbar \nu_{F} K}{E-V+m \nu_{\mathrm{F}}^{2}}
$$

The transfer matrix for a given region (extending between two matching points $x_{1}$ and $x_{2}$ specifying the potential steps $\left.m_{i}^{(n)}\right)$ is the product $\mathbf{M}_{K}\left(x_{1}, x_{2}\right)=\boldsymbol{\Omega}_{K}\left(x_{2}\right) \boldsymbol{\Omega}_{K}^{-1}\left(x_{1}\right)$; this latter matrix depends only on the width $x_{2}-x_{1}$ of the region and not separately on $x_{1}$ or $x_{2}$.

The transfer matrix corresponding to a series of $N$ regions can be formed ${ }^{21}$ as the product

$$
\mathbf{t}_{1, N+1}=\prod_{i=1, N} \mathbf{M}_{K_{i}}\left(x_{i}, x_{i+1}\right)
$$

where $\left|x_{i+1}-x_{i}\right|=L_{i}$ is the width of the $i$ th region [with $\left.(m, V, K, \Lambda) \rightarrow\left(m_{i}, V_{i}, K_{i}, \Lambda_{i}\right)\right]$. The transfer matrix associated with the transmission of a free Fermion of mass $\mathcal{M}$ (incoming from the right) through the multiple mass barriers is the product

$$
\mathcal{T}(E)=\boldsymbol{\Omega}_{k}^{-1}\left(x_{N+1}\right) \mathbf{t}_{1, N+1} \boldsymbol{\Omega}_{k}\left(x_{1}\right)
$$

with $k=\left[(E-V)^{2}-\mathcal{M}^{2} \nu_{\mathrm{F}}^{4}\right]^{1 / 2} /\left(\hbar \nu_{\mathrm{F}}\right),|E-V| \geq \mathcal{M} \nu_{\mathrm{F}}^{2}$; for armchair leads, $V=0$, while for zigzag leads $V=\mp \mathcal{M} \nu_{\mathrm{F}}^{2}$. Naturally, in the case of metallic armchair leads, $k=E /\left(\hbar \nu_{\mathrm{F}}\right)$ because $\mathcal{M}=0$.

Then, the transmission coefficient $T$ is

$$
T=\frac{1}{\left|\mathcal{T}_{22}\right|^{2}}
$$

while the reflection coefficient is given by

$$
R=\left|\frac{\mathcal{T}_{12}}{\mathcal{T}_{22}}\right|^{2}
$$

At zero temperature, the conductance is given by $G=\left(2 e^{2}\right)$ h) $T$; $T$ is the transmission coefficient in eq 6 .

DKP Superlatice Model. The transfer matrix corresponding to either half of the rhombus can be formed ${ }^{21}$ as the product

$$
\mathbf{t}_{n}=\prod_{i=1,3} \mathbf{M}_{K}\left(x_{i}, x_{i+1}\right) \quad x_{1}=0 \quad x_{4}=L
$$




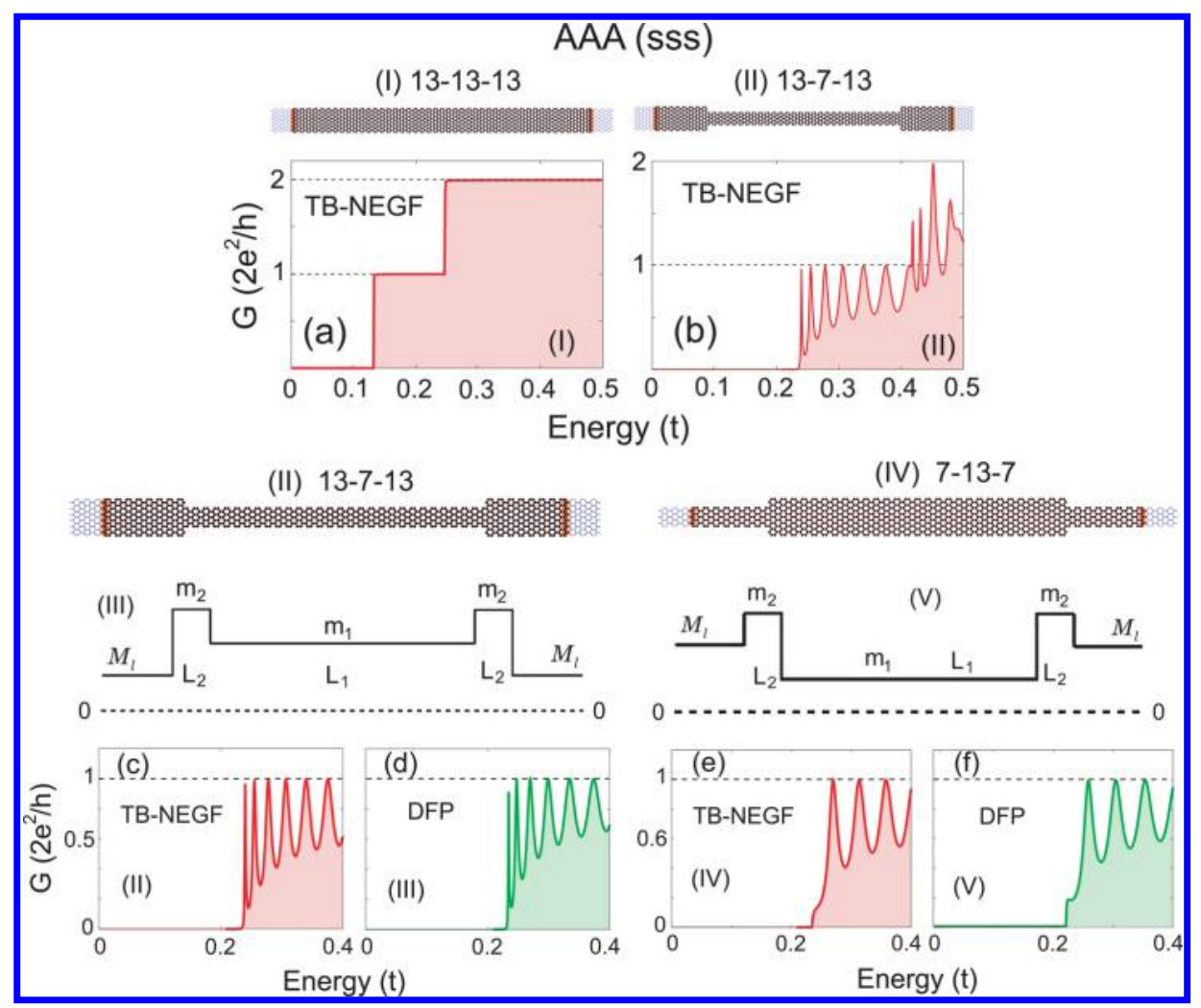

Figure 1. Conductance quantization steps (a) for a uniform semiconducting armchair nanoribbon (I) contrasted to FP oscillations (b-f) of two three-segment aGNRs [(II) and (IV)] with both a semiconducting central constriction and semiconducting leads (13-7-13 and 7-13-7). For the systems shown here, all of the segments have armchair edge termination (hence, AAA), and all have widths corresponding to semiconducting GNRs [hence (sss)]. (III,V) Schematics of the mass barriers used in the DFP modeling, with the dashed line denoting the zero mass. The physics underlying such a junction is that of a massive relativistic Dirac Fermion impinging upon the junction and performing multiple reflections (above $m_{1} \nu_{\mathrm{F}}^{2}$ ) within a particle box defined by the double-mass barrier. (c,e) TB-NEGF conductance as a function of the Fermi energy of the massive Dirac electrons in the leads. (d) DFP conductance reproducing [in the energy range of the $1 G_{0}$ step; see (b)] the TB-NEGF result in (c). The mass barrier parameters used in the DFP reproduction were $L_{1}=55 a_{0}, m_{1} \nu_{\mathrm{F}}^{2}=0.22 t, L_{2}=1 a_{0}$, and $m_{2} \nu_{\mathrm{F}}^{2}=0.5 t$. The mass of the electrons in the leads was $\mathcal{M}_{1} \nu_{\mathrm{F}}^{2}=$ 0.166t. (f) DFP conductance reproducing the TB-NEGF result in (e). The parameters used in the DFP reproduction were $L_{1}=53.6 a_{0}, m_{1} \nu_{\mathrm{F}}^{2}=$ $0.166 t, L_{2}=1 a_{0}$, and $m_{2} \nu_{\mathrm{F}}^{2}=0.51$. The mass of the electrons in the leads was $\mathcal{M}_{1} \nu_{\mathrm{F}}^{2}=0.22 t . a_{0}=0.246 \mathrm{~nm}$ is the graphene lattice constant; $t=2.7 \mathrm{eV}$ is the hopping parameter.

with $L$ being the length of half of the perimeter of the rhombus; $L=L_{1}+L_{2}+L_{3}$, with $L_{1}=L_{3}=a$ and $L_{2}=b$. The transfer matrix associated with the complete unit cell (encircling the rhombic ring) is the product

$$
\mathbf{T}=\prod_{n=1}^{2} \mathbf{t}_{n}
$$

Following refs 21 and 52, we consider the superlattice generated from the virtual periodic translation of the unit cell as a result of the application of a magnetic field $B$ perpendicular to the ring. Then, the $\mathrm{AB}$ energy spectra are given as solutions of the dispersion relation

$$
\cos \left[2 \pi\left(\frac{\Phi}{\Phi_{0}}+\eta\right)\right]=\frac{\operatorname{Tr}[\mathbf{T}(E)]}{2}
$$

where we have explicitly denoted the dependence of the righthand side on the energy $E ; \eta=0$ for a rhombus with type-I corners, and $\eta=1 / 2$ for a rhombus with type-II corners.

The energy spectra and single-particle densities do not depend on a specific representation. However, the wave functions (upper and lower spinor components of the Fermionic field $\Psi$ ) do depend on the representation used. To transform the initial DKP wave functions to the $\left(\alpha=\sigma_{2}, \beta=\right.$ $\left.\sigma_{1}\right)$ representation, which corresponds to the natural separation of the TB amplitudes into the $A$ and $B$ sublattices, we apply successively the unitary transformations $D_{23}=\left(\sigma_{2}+\sigma_{3}\right) / 2^{1 / 2}$ and $D_{3}=\exp \left(\mathrm{i} \pi \sigma_{3} / 4\right)$.

TB-NEGF Formalism. To describe the properties of graphene nanostructures in the TB approximation, we use the Hamiltonian

$$
H_{\mathrm{TB}}=-t \sum_{\langle i, j\rangle} c_{i}^{\dagger} c_{j}+\text { h.c. }
$$

with \langle\rangle indicating summation over the nearest-neighbor sites $i, j . t=2.7 \mathrm{eV}$ is the hopping parameter of $2 \mathrm{D}$ graphene.

To calculate the TB-NEGF transmission coefficients, the Hamiltonian in eq 11 is employed in conjunction with the wellknown transport formalism, which is based on the nonequilibrium Green's functions. ${ }^{40,41}$

According to the Landauer theory, the linear conductance is $G(E)=\left(2 e^{2} / h\right) T(E)$, where the transmission coefficient is calculated as $T(E)=\operatorname{Tr}\left[\Gamma_{\mathrm{L}} \mathcal{G} \Gamma_{\mathrm{R}} \mathcal{G}^{\dagger}\right]$. The Green's function $\mathcal{G}(E)$ is given by

$$
\mathcal{G}(E)=\left(E+\mathrm{i} \eta-H_{\mathrm{TB}}^{\mathrm{dev}}-\Sigma_{\mathrm{L}}-\Sigma_{\mathrm{R}}\right)^{-1}
$$


with $H_{\mathrm{TB}}^{\mathrm{dev}}$ being the Hamiltonian of the isolated device (junction without the leads). The self-energies $\Sigma_{\mathrm{L}(\mathrm{R})}$ are given by $\Sigma_{\mathrm{L}(\mathrm{R})}=\tau_{\mathrm{L}(\mathrm{R})}\left[E+\mathrm{i} \eta-H_{\mathrm{TB}}^{\mathrm{L}(\mathrm{R})}\right]^{-1} \tau_{\mathrm{L}(\mathrm{R})}^{\dagger}$, where the hopping matrixes $\tau_{\mathrm{L}(\mathrm{R})}$ describe the left (right) device-to-lead coupling and $H_{\mathrm{TB}}^{\mathrm{L}(\mathrm{R})}$ is the Hamiltonian of the semi-infinite left (right) lead. The broadening matrices are given by $\Gamma_{\mathrm{L}(\mathrm{R})}=\mathrm{i}\left[\Sigma_{\mathrm{L}(\mathrm{R})}-\right.$ $\left.\Sigma_{\mathrm{L}(\mathrm{R})}^{\dagger}\right]$.

\section{RESULTS}

Segmented aGNRs: All-Semiconducting. Our results for a three-segment $\left(\mathcal{N}_{1}^{W}-\mathcal{N}_{2}^{W}-\mathcal{N}_{1}^{W}\right.$, where $\mathcal{N}_{1}^{W}$ is the lead width and $\mathcal{N}_{2}^{W}$ is the width of the central segment) all-semiconducting aGNR, AAA (sss), are portrayed in Figure 1 [see schematic lattice diagrams in Figure 1I and II]. A uniform semiconducting aGNR [see Figure 1I] exhibits ballistic quantized conductance steps [see Figure 1a]. In contrast, conductance quantization is absent for a nonuniform threesegment (13-7-13) aGNR; see Figure 1b-d. Here, oscillations appear instead of quantized steps. The first oscillation appears at an energy of $\sim 0.22 t$ [Figure $1 \mathrm{~b}$ ], which reflects the intrinsic gap $\Delta / 2$ of the semiconducting central segment belonging to the class II of aGNRs, specified ${ }^{10,13,22}$ by a width $\mathcal{N}^{W}=3 l+1$, with $l=1,2,3, \ldots$ We recall that as a function of their width, $\mathcal{N}^{W}$, the aGNRs fall into three classes: (I) $\mathcal{N}^{W}=3 l$ (semiconducting, $\Delta>0$ ), (II) $\mathcal{N}^{W}=3 l+1$ (semiconducting, $\Delta>0$ ), and (III) $\mathcal{N}^{W}=3 l+2$ (metallic, $\Delta$ $=0), l=1,2,3, \ldots$

That the leads are semiconducting does not have any major effect. This is due to the fact that $\mathcal{N}_{2}^{W}<\mathcal{N}_{1}^{W}$, and as a result, the energy gap $m_{1} \nu_{\mathrm{F}}^{2}$ of the central segment is larger than the energy gap $\mathcal{M}_{l} \nu_{\mathrm{F}}^{2}$ of the semiconducting leads [see the schematic in Figure 1III]. In the opposite case (central segment wider than the leads), the energy gap of the semiconducting leads would have determined the onset of the conductance oscillations.

The aGNR case with interchanged widths (i.e., 7-13-7 instead of 13-7-13) is portrayed in Figure 1e-f. In this case, the energy gap of the semiconducting leads (being the largest) determines the onset of the conductance oscillations. It is a testimonial of the consistency of our DFP method that it can reproduce [see Figure $1 \mathrm{~d}$ and $\mathrm{f}$ ] both the $13-7-13$ and 7-137 TB-NEGF conductances; this is achieved with very similar sets of parameters, taking into consideration the centralsegment-leads interchange. We note that the larger spacing between peaks (and also the smaller number of peaks) in the $7-13-7$ case is due to the smaller mass of the central segment (0.166t instead of $0.22 t$ ).

Further insight can be gained by an analysis of the discrete energies associated with the humps of the conductance oscillations in Figure 1c and the resonant spikes in Figure 1e. Indeed, a simplified approximation for the electron confinement in the continuum model consists of considering the graphene electrons as being trapped within a $1 \mathrm{D}$ infinite mass square well (IMSW) of length $L_{1}$ (the mass terms are infinite outside of the interval $L_{1}$, and the coupling to the leads vanishes). The discrete spectrum of the electrons in this case is given $^{53}$ by

$$
E_{n}=\sqrt{\hbar^{2} \nu_{\mathrm{F}}^{2} k_{n}^{2}+\mathcal{M}^{2} \nu_{\mathrm{F}}^{4}}
$$

where the wave numbers $k_{n}$ are solutions of the transcendental equation

$$
\tan \left(k_{n} L_{1}\right)=\frac{-\hbar k_{n}}{\left(\mathcal{M} \nu_{\mathrm{F}}\right)}
$$

When $\mathcal{M}=0$ [massless DW electrons], one finds for the spectrum of the IMSW model

$$
E_{n}=\frac{\left(n+\frac{1}{2}\right) \pi \hbar \nu_{\mathrm{F}}}{L_{1}}
$$

with $n=0,1,2, \ldots$

$$
E_{n}-E_{n-1}=2 E_{0} \quad n=1,2,3, \ldots
$$

which is twice the energy

$$
E_{0}=\frac{\pi \hbar \nu_{\mathrm{F}}}{\left(2 L_{1}\right)}
$$

of the lowest state.

As is well-known, a constant energy separation of the intensity peaks, inversely proportional to the length of the resonating cavity [here $L_{1}$; see eqs 16 and 17], is the hallmark of the OFP, reflecting the linear energy dispersion of the photon in optics ${ }^{38}$ or a massless DW electron in graphene structures. ${ }^{36}$ Most revealing is the energy offset away from zero of the first conductance peak, which equals exactly one-half of the constant energy separation between the peaks. In onedimension, this is the hallmark of a massless Fermion subject to an infinite mass barrier confinement. ${ }^{53}$ Naturally, in the case of a semiconducting segment (see below), this equidistant behavior and half-offset of the conductance peaks do not apply; this case is accounted for by the DFP model presented in the Methods Section, and it is more general than the OFP theory associated with a photonic cavity. ${ }^{38}$

In the nonrelativistic limit, that is, when $\hbar k_{n} \ll \mathcal{M} \nu_{\mathrm{F}}$, one gets

$$
\tan \left(k_{n} L_{1}\right) \approx 0
$$

which yields the well-known relations $k_{n} L_{1} \approx n \pi$ and

$$
E_{n} \sim \mathcal{M} \nu_{\mathrm{F}}^{2}+\frac{n^{2} \hbar^{2} \pi^{2}}{\left(2 \mathcal{M} L_{1}^{2}\right)}
$$

For a massive relativistic electron, as is the case with the semiconducting aGNRs in this paper, one has to numerically solve eq 14 and then substitute the corresponding value of $k_{n}$ in Einstein's energy relation given by eq 13 .

From an inspection of Figure 1, one can conclude that the physics associated with the all-semiconducting AAA junction is that of multiple reflections of a massive relativistic Dirac Fermion bouncing back and forth from the edges of a "quantum box" created by a double-mass barrier [see the schematic of the double-mass barrier in Figures $1 \mathrm{III}$ and V]. In particular, to a good approximation, the energies of the conductance oscillation peaks are given by the IMSW (eq 14) with $\mathcal{M}=m_{1} \nu_{\mathrm{F}}^{2}=0.22 t(13-7-13)$ or $\mathcal{M}=m_{1} \nu_{\mathrm{F}}^{2}=0.166 t$ (713-7). In this respect, the separation energy between successive peaks in Figure $1 \mathrm{~b}, \mathrm{c}$, e, and $\mathrm{f}$ is not a constant, unlike the case of an all-metallic junction ${ }^{36}$ (or a photonic cavity $^{38}$ ).

The conductance patterns in Figure $1 \mathrm{~d}$ and $\mathrm{f}$ correspond to the category FP-B (see the Introduction). These patterns cannot be accounted for by the OFP theory, but they are well 


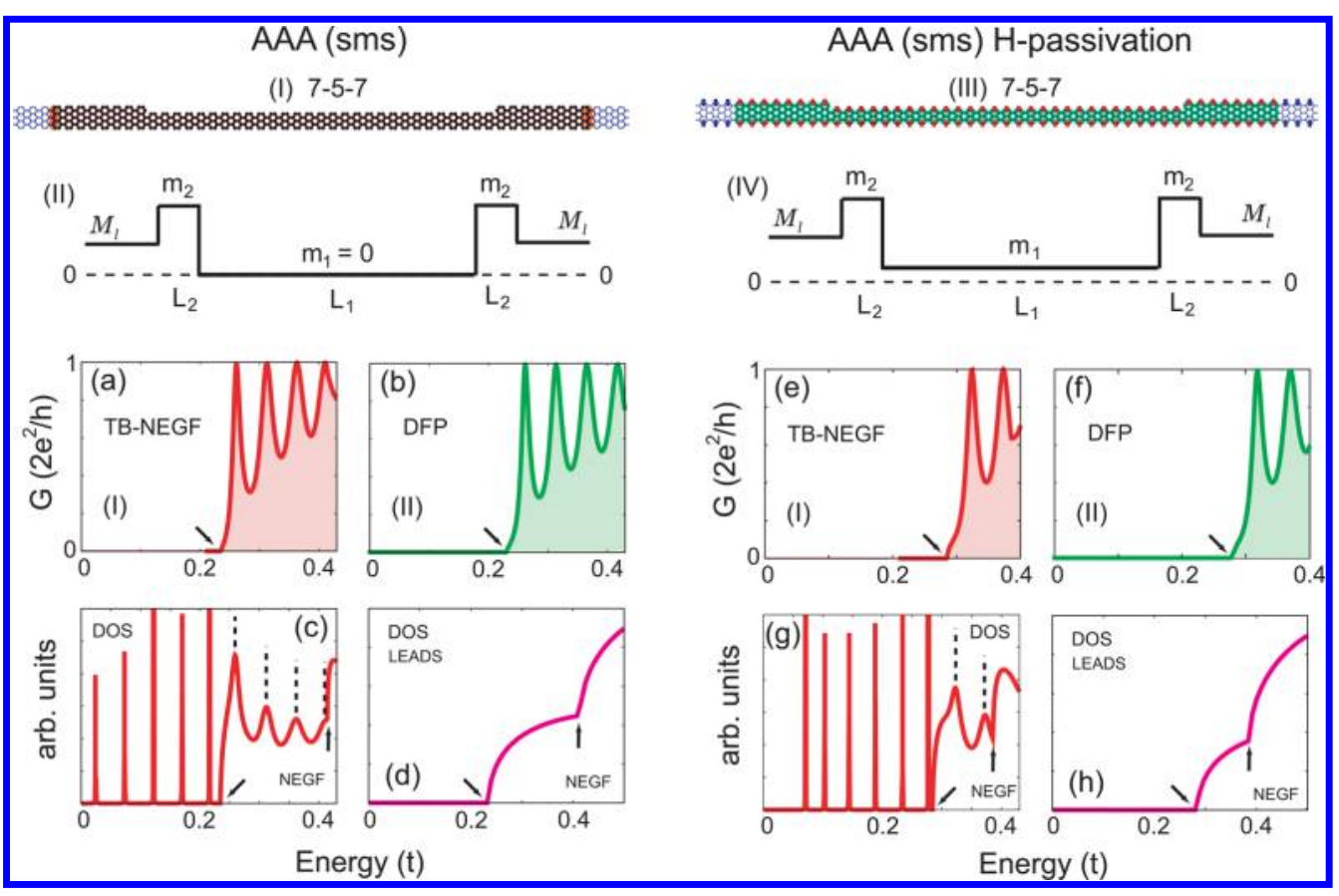

Figure 2. (Left) Conductance for a three-segment nanoribbon, all segments having armchair edge termination (i.e., $\mathrm{AAA})$, with a metallic $\left(\mathcal{N}_{2}^{W}=5\right)$ central constriction and semiconducting leads $\left(\mathcal{N}_{1}^{W}=7\right)$ and hence the designation (sss); see the schematic lattice diagram in (I). (II) Schematics of the mass barriers used in the DFP modeling. (a) TB-NEGF conductance as a function of the Fermi energy of the massive Dirac electrons in the leads. (b) DFP conductance reproducing the TB-NEGF result in (a). The mass barrier parameters used in the DFP reproduction were $L_{1}=60.4 a_{0}$, $m_{1}=0, L_{2}=1 a_{0}$, and $m_{2} \nu_{\mathrm{F}}^{2}=0.37 t$. The mass of the electrons in the leads was $\mathcal{M}_{1} \nu_{\mathrm{F}}^{2}=0.23 t$. (c,d) The total DOS of the junction and the density of states in the isolated leads, respectively, according to the TB-NEGF calculations. The arrows indicate the onset of the electronic bands in the leads. Note that the DOS in (c) reveals the existence of five sharp electronic states below the onset (at $\left.0.23 t \equiv \mathcal{M}_{l} \nu_{\mathrm{F}}^{2}\right)$ of the first band in the leads [see (d)], which consequently do not generate any conductance resonances [see (a) and (b)]. Note further in (c) the equal energy spacing between the vertical lines [the five solid (red) and four dashed (black) ones] associated with the resonances of a massless electron confined within the central metallic aGNR segment. (Right) H-passivation effects in the conductance of a three-segment armchair nanoribbon with a metallic $\left(\mathcal{N}_{2}^{W}=5\right)$ central constriction and semiconducting leads $\left(\mathcal{N}_{1}^{W}=7\right)$; see the schematic lattice diagram in (III). Note that the nearest-neighbor $\mathrm{C}-\mathrm{C}$ bonds at the armchair edges (thick red and blue lines) have hopping parameters $t^{\prime}=1.12 t$. (IV) Schematics of the position-dependent mass field used in the DFP modeling. (e) TB-NEGF conductance as a function of the Fermi energy. (f) DFP conductance reproducing the TB-NEGF result in (e). The mass parameters used in the DFP reproduction were $L_{1}=59.5 a_{0}, m_{1} \nu_{\mathrm{F}}^{2}=0.05 t, L_{2}=1.5 a_{0}$, and $m_{2} \nu_{\mathrm{F}}^{2}=0.30 t$. The mass of the electrons in the leads was $\mathcal{M}_{l} \nu_{\mathrm{F}}^{2}=0.28 t .(\mathrm{g}, \mathrm{h})$ The total DOS of the junction and the DOS in the isolated leads, respectively, according to the TB-NEGF calculations. The arrows indicate the onset of the electronic bands in the leads; note the shifts from $0.23 t$ to $0.28 t$ and from $0.42 t$ to $0.38 t$ for the onsets of the first and second bands, respectively, compared to the case with $t^{\prime}=t$ in (d). Compared to the left part, the subtle modifications of mass parameters brought about by having $t^{\prime}=1.12 t$ result in having six sharp electronic states [see $\left.(\mathrm{g})\right]$ below the onset $\left(\right.$ at $\left.0.28 t \equiv \mathcal{M}_{l} \nu_{\mathrm{F}}^{2}\right)$ of the first band in the leads [see (h)], which consequently do not generate any conductance resonances [see (e) and (f)]. In addition, within the energy range (0t-0.4t) shown in (e) and (f), there are now only two conducting resonances, instead of three compared to (a) and (b). $a_{0}=0.246 \mathrm{~nm}$ is the graphene lattice constant; $t=$ $2.7 \mathrm{eV}$ is the graphene hopping parameter.

reproduced by the generalized DFP model introduced by us in the Methods Section.

Segmented aGNRs: Semiconducting-Metallic-Semiconducting. Our results for a three-segment (7-5-7) semiconducting-metallic-semiconducting aGNR, AAA (sms), are portrayed in Figure 2 [left; see the schematic lattice diagram in Figure 2I]. The first FP oscillation in the TB-NEGF conductance displayed in Figure 2a appears at an energy of $\sim 0.22 t$, which reflects the intrinsic gap $\Delta / 2$ of the semiconducting leads (with $\mathcal{N}_{1}^{W}=7$ ). The energy spacing between the peaks in Figure 2a is constant, in agreement with the metallic (massless DW electrons) character of the central segment with $\mathcal{N}_{2}^{W}=5$. The TB-NEGF pattern in Figure 2a corresponds to the FP category FP-A2. As seen from Figure $2 b$, our generalized DFP theory is again capable of faithfully reproducing this behavior.
A deeper understanding of the AAA (sms) case can be gained via an inspection of the density of states (DOS) plotted in Figure $2 \mathrm{c}$ for the total segmented aGNR (central segment plus leads) and in Figure $2 \mathrm{~d}$ for the isolated leads. In Figure 2c, nine equidistant resonance lines are seen. Their energies are close to those resulting from the IMSW eq 15 (with $L_{1}=60.4 a_{0}$; see the caption of Figure 2) for a massless DW electron. Out of these nine resonances, the first five do not conduct [compare Figure $2 \mathrm{a}$ and $\mathrm{c}$ ] because their energies are lower than the minimum energy (i.e., $\Delta / 2=\mathcal{M}_{l} \nu_{\mathrm{F}}^{2} \approx 0.23 t$ ) of the incoming electrons in the leads [see the onset of the first band (marked by an arrow) in the DOS curve displayed in Figure 2d].

Segmented aGNRs: Effects of Hydrogen Passivation. As shown in refs 11 and 12, a detailed description of hygrogen passivation requires that the hopping parameters $t^{\prime}$ for the nearest-neighbor $\mathrm{C}-\mathrm{C}$ bonds at the armchair edges be given by $t^{\prime}=1.12 t$. Taking this modification into account, our results for a three-segment semiconducting-metallic-semiconducting 
aGNR are portrayed in Figure 2 [right; see the schematic lattice diagram in Figure 2II)]; this lattice configuration is denoted as "AAA (sms) H-passivation". The first FP oscillation in the TBNEGF conductance displayed in Figure 2e appears at an energy of $\sim 0.28 t$, which reflects the intrinsic gap $\Delta / 2$ of the properly passivated semiconducting leads (with $\mathcal{N}_{1}^{W}=7$ ). The energy spacing between the peaks in Figure $2 \mathrm{e}$ is slightly away from being constant in agreement with the small mass $m_{1} \nu_{F}^{2}=0.05 t$ acquired by the central segment with $\mathcal{N}_{2}^{W}=5$ due to taking $t^{\prime}$ $=1.12 t$. As seen from Figure 2f, our generalized DFP theory is again capable of faithfully reproducing this behavior.

A deeper understanding of the AAA (sms) H-passivation case can be gained via an inspection of the DOS plotted in Figure $2 \mathrm{~g}$ for the total segmented aGNR (central segment plus leads) and in Figure $2 \mathrm{~h}$ for the isolated leads. In Figure $2 \mathrm{~g}$, eight (almost, but not exactly, equidistant) resonance lines are seen. Their energies are close to those resulting from the IMSW eq 14 (with $L_{1}=59.5 a_{0}$ and $m_{1} \nu_{\mathrm{F}}^{2}=0.05 t$; see the caption of Figure 2) for a Dirac electron with a small mass. Out of these eight resonances, the first six do not conduct [compare Figure $2 \mathrm{e}$ and $\mathrm{g}$ ] because their energies are lower than the minimum energy (i.e., $\Delta / 2=\mathcal{M}_{l} \nu_{\mathrm{F}}^{2} \approx 0.28 t$ ) of the incoming electrons in the leads [see the onset of the first band (marked by an arrow) in the DOS curve displayed in Figure 2h]. From the above, we conclude that hydrogen passivation of the aGNR resulted in a small shift of the location of the states and opening of a small gap for the central metallic narrower (with a width of $\mathcal{N}_{2}^{W}=5$ ) segment but did not modify the conductance record in any qualitative way. Moreover, the passivation effect can be faithfully captured by the Dirac FP model by a small readjustment of the model parameters.

All-Zigzag Segmented GNRs. It is interesting to investigate the sensitivity of the interference features on the edge morphology. We show in this section that the relativistic transport treatment applied to segmented armchair GNRs does not maintain for the case of a nanoribbon segment with zigzag edge terminations. In fact, zGNR segments exhibit properties akin to the well-known transport in usual semiconductors, that is, their excitations are governed by the nonrelativistic Schrödinger equation.

Before discussing segmented GNRs with zigzag edge terminations, we remark that such GNRs with uniform width exhibit stepwise quantization of the conductance, similar to the case of a uniform armchair-edge-terminated GNR (see Figure 1a).

In Figure 3a, we display the conductance in a three-segment junction (see the lattice schematic in Figure 3I) when all three segments have zigzag edge terminations (denoted as ZZZ) but the central one is narrower than the lead segments. The main finding is that the central segment behaves again as a resonant cavity that yields an oscillatory conductance pattern where the peak spacings are unequal (Figure 3a). This feature, which deviates from the OFP behavior, appeared also in the DFP patterns for a three-segment armchair junction whose central segment was semiconducting, albeit with a different dependence on $L$ (see Figure 1 and eq 14). Moreover, from a set of systematic calculations (not shown) employing different lengths and widths, we found that the energy of the resonant levels in zGNR segments varies on averag as $\sim(n / L)^{2}$, where the integer $n$ counts the resonances and $L$ indicates the length of the central segment. However, a determining difference with the aGNR case in Figure 1 is the vanishing of the valence-to-

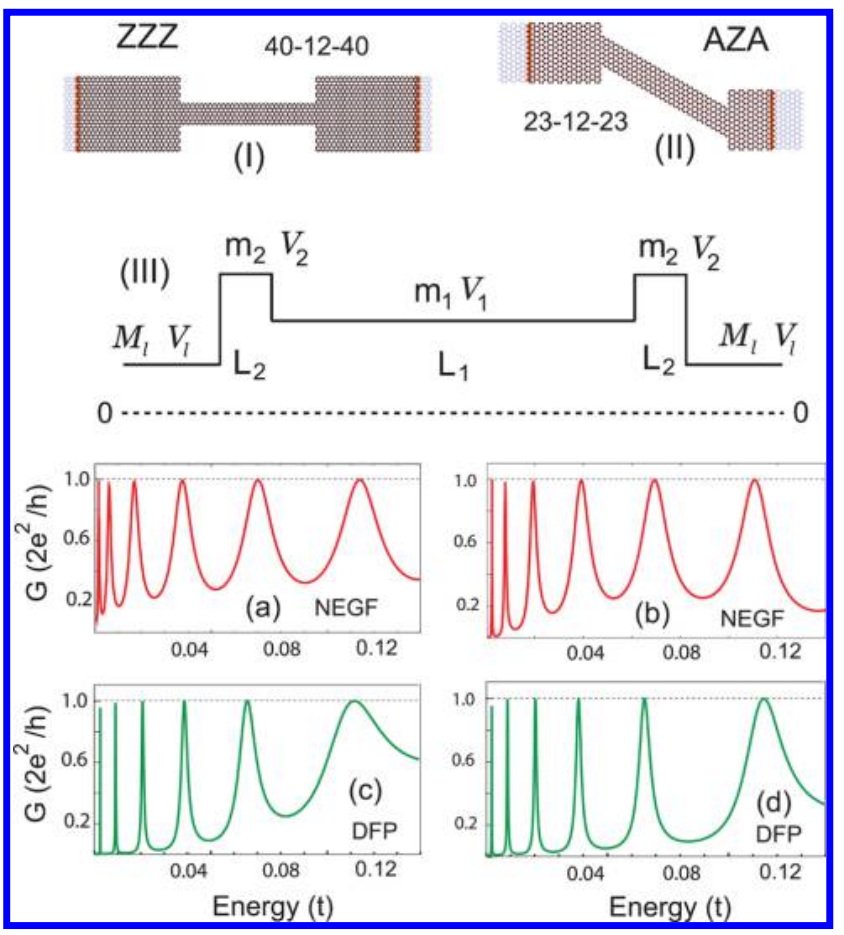

Figure 3. Conductance for ZZZ (all-zigzag edge termination, left column) and AZA (armchair-zigzag-armchair edge termination, right column) segmented nanoribbon junctions. See the corresponding lattice diagrams in (I) and (II). The three-segment GNRs are denoted as $\mathcal{N}_{1}^{W}-\mathcal{N}_{2}^{W}-\mathcal{N}_{1}^{W}$, with $\mathcal{N}_{i}^{W}(i=1,2)$ being the number of carbon atoms specifying the width of the ribbon segments. The armchair leads in the AZA junction are metallic $\left(\mathcal{N}_{1}^{W}=23\right.$, class III aGNR). (a,b) TB-NEGF conductance for the ZZZ and AZA junctions, respectively. (c) DFP conductance reproducing the TB-NEGF result in (a) for the ZZZ junction. (d) DFP conductance reproducing the TB-NEGF result in (b) for the AZA junction. Despite the different edge morphology, the FP patterns in (a) and (b) are very similar. The central zigzag segment controls the FP patterns. According to the continuum DFP analysis, the physics underlying such patterns is that of a massive nonrelativistic Schrödinger Fermionic carrier performing multiple reflections within a cavity defined by a double-mass barrier [see diagram in (III)] but with the additional feature that $V_{1}=-m_{1} \nu_{\mathrm{F}}^{2}$ and $V_{l}=-\mathcal{M}_{l} \nu_{\mathrm{F}}^{2}$ are also considered for segments or leads with zigzag edge terminations (see the text for details). The mass and $V_{i}$ parameters used in the DFP calculations were $L_{1}=30 a_{0}, m_{1} \nu_{\mathrm{F}}^{2}=2.23 t-c E t$, with $c$ $=7.3, V_{1}=-m_{1} \nu_{\mathrm{F}}^{2}, L_{2}=1.1 a_{0}, m_{2} \nu_{\mathrm{F}}^{2}=0.38 t, V_{2}=-m_{2} \nu_{\mathrm{F}}^{2} / 3, \mathcal{M}_{l} \nu_{\mathrm{F}}^{2}=$ 2.30t, and $V_{l}=-\mathcal{M}_{l} \nu_{\mathrm{F}}^{2}$ in (c) and $L_{1}=29.1 a_{0}, m_{1} \nu_{\mathrm{F}}^{2}=2.65 t-c E t$, with $c=8.4, V_{1}=-m_{1} \nu_{\mathrm{F}}^{2}, L_{2}=1.0 a_{0}, m_{2} \nu_{\mathrm{F}}^{2}=0.30 t, V_{2}=-m_{2} \nu_{\mathrm{F}}^{2}, \mathcal{M}_{l}=0$, and $V_{l}=0$ in (d). $\mathcal{M}_{l}$ and $V_{l}$ denote parameters of the leads. $E$ is the energy in units of $t$. $a_{0}=0.246 \mathrm{~nm}$ is the graphene lattice constant; $t=$ $2.7 \mathrm{eV}$ is the hopping parameter.

conductance gap in the zigzag case of Figure 3a. It is wellknown that the above features are associated with resonant transport of electronic excitations that obey the nonrelativistic second-order Schrödinger equation.

Naturally, one could formulate a continuum transport theory based on transfer matrices (see the Methods Section) that use the 1D Schrödinger equation instead of the generalized Dirac eq 1. Such a Schrödinger equation continuum approach, however, is unable to describe mixed armchair-zigzag interfaces (see below), where the electron transits between two extreme regimes, that is, an ultrarelativistic (i.e., including the limit of vanishing carrier mass) Dirac regime (armchair segment) and a nonrelativistic Schrödinger regime (zigzag 


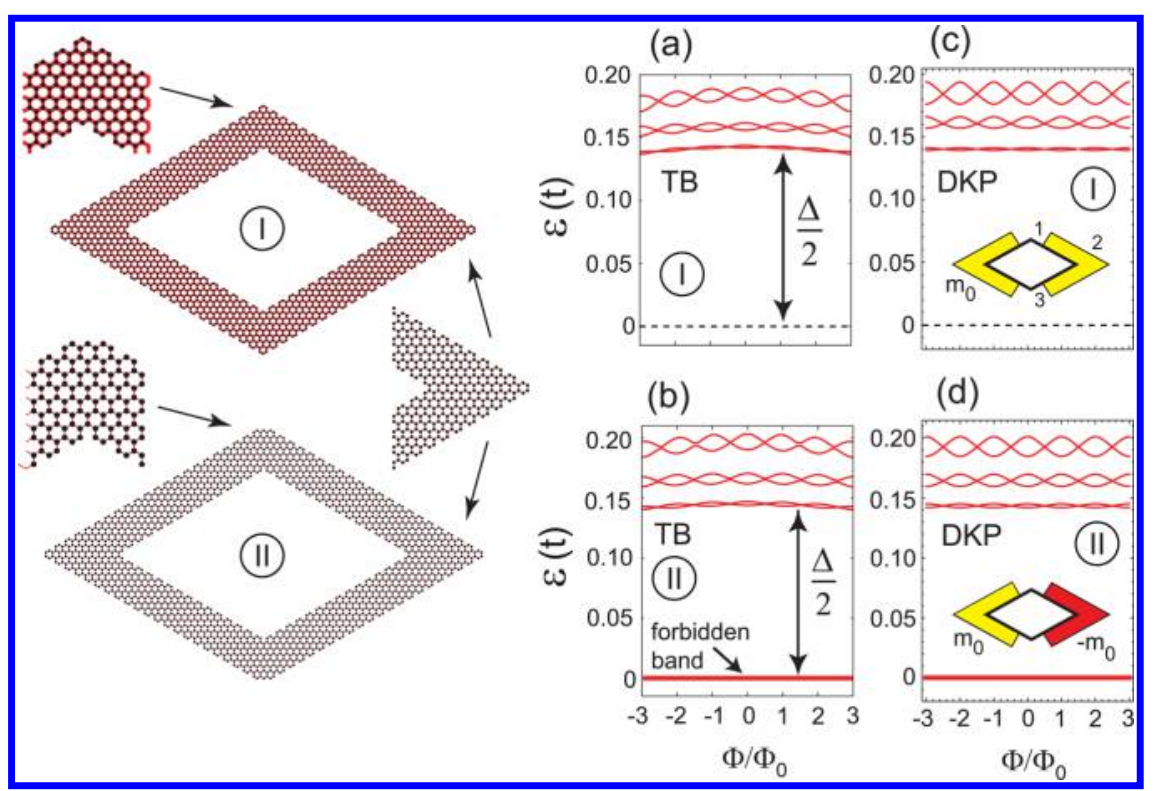

Figure 4. $\mathrm{AB}$ spectra for rhombic AGRs; the two rings that we consider (I and II on the left) show different atomic arrangements at the top and bottom corners. (a) TB spectrum for a nanoring with type-I corners and width $\mathcal{N}^{W}=12$. (b) TB spectrum for a nanoring with type-II corners and the same width $\mathcal{N}^{W}=12$. These AGRs are semiconducting (type-I) and metallic (type-II). The three (four) lowest-in-energy two-membered bands are shown. The hole states (with $\varepsilon<0$, not shown) are symmetric to the particle states (with $\varepsilon>0$ ). (c,d) DKP spectra reproducing the TB ones in (a) and (b), respectively. Insets in (c) and (d): schematics of the Higgs fields (position-dependent mass) $\phi(x)$ employed in the DKP modeling. $\phi(x)$ is approximated by steplike functions $m_{i}{ }^{(n)} ; i$ counts the three regions of each half of the rhombus $\left(L_{1}{ }^{(n)}=L_{3}{ }^{(n)}=a\right.$ and $\left.L_{2}{ }^{(n)}=b\right)$, and $n(n=1,2)$. The nonzero (constant) variable mass values of $\phi(x)$ are indicated by yellow (red) color when positive (negative). The parameters used in the DKP modeling are (c) $a=1.3 a_{0}, b=66 a_{0}, m_{1}{ }^{(n)}=m_{3}{ }^{(n)}=0$, and $m_{2}{ }^{(n)}=m_{0}=0.15 t / \nu_{\mathrm{F}}^{2}$ [see the corresponding schematic inset in (c)] and (d) $a=6.5 a_{0}, b$ $=55 a_{0}, m_{1}{ }^{(n)}=m_{3}{ }^{(n)}=0, m_{2}{ }^{(n)}=(-1)^{n} m_{0}$, with $m_{0}=0.15 t / \nu_{\mathrm{F}}^{2}$ [see the schematic inset in (d)]. Note the two-membered braided bands and the "forbidden" band [within the gap, in (b) and (d)]. The two-fold forbidden band with $\epsilon \approx 0$ appears as a straight line due to the very small amplitude oscillations of its two members. $a_{0}=0.246 \mathrm{~nm}$ is the graphene lattice constant, and $t=2.7 \mathrm{eV}$ is the hopping parameter. The edge terminations of both the inside and outside sides of the ring are armchair.

segment). We have thus been led to adopt the same Dirac-type transfer matrix approach as with the aGNRs but with nonvanishing potentials $V=\mp \mathcal{M} \nu_{\mathrm{F}}^{2}$. This amounts to shifts (in opposite senses) of the energy scales for particle and hole excitations, respectively, and it yields the desired vanishing value for the valence-to-conduction gap of zGNRs.

The calculated DFP conductance that reproduces well the TB-NEGF result for the ZZZ junction (Figure 3a) is displayed in Figure 3c; the parameters used in the DFP calculation are given in the caption of Figure 3. We note that the carrier mass $\left(m_{1}\right)$ in the central zigzag segment exhibits an energy dependence. This is similar to a well-known effect (due to nonparabolicity in the $E-k$ dispersion) in the transport theory of usual semiconductors. ${ }^{54}$ We further note that the average mass associated with a zigzag segment is an order of magnitude larger than that found for semiconducting armchair segments of similar width (see the caption in Figure 1), and this yields energy levels of $\sim(n / L)^{2}$, close to the nonrelativistic limit (see eq 19). We note that the FP pattern of the ZZZ junction belongs to the category FP-C.

Mixed Armchair-Zigzag-Armchair Segmented GNRs. Figure 3 (right column) presents an example of a mixed armchair-zigzag-armchair (AZA) junction, where the central segment has again zigzag edge terminations (see the lattice schematic in Figure 3II). The corresponding TB-NEGF conductance is displayed in Figure $3 \mathrm{~b}$. Despite the different morphology of the edges between the leads (armchair) and the central segment (zigzag), the conductance profile of the AZA junction (Figure $3 \mathrm{~b}$ ) is very similar to that of the $\mathrm{ZZZ}$ junction (Figure 3a). This means that the characteristics of the transport are determined mainly by the central segment, with the left and right leads, whether zigzag or armchair, acting as reservoirs supplying the impinging electrons.

The DFP result reproducing the TB-NEGF conductance is displayed in Figure 3d, and the parameters used are given in the caption. We stress that the mixed AZA junction represents a rather unusual physical regime, where an ultrarelativistic DW massless charge carrier (due to the metallic aGNRs in the leads) transits to a nonrelativistic massive Schrödinger electron in the central segment. We note that the FP pattern of the AZA junction belongs to the FP-C caregory.

AB Spectra of Rhombic Graphene Rings. The energy of a particle (with $1 \mathrm{D}$ momentum $p_{x}$ ) is given by the Einstein relativistic relation $E=\sqrt{\left(p_{x} v_{\mathrm{F}}\right)^{2}+\left(\mathcal{M} v_{\mathrm{F}}^{2}\right)^{2}}$, where $\mathcal{M}$ is the rest mass. As aforementioned, in armchair graphene ribbons, the mass parameter is related to the particle-hole energy gap, $\Delta$, as $\mathcal{M}=\Delta /\left(2 \nu_{\mathrm{F}}^{2}\right)$. In relativistic quantum field theory, the mass of elementary particles is imparted through interaction with a scalar field known as the Higgs field. Accordingly, the mass $\mathcal{M}$ is replaced by a position-dependent Higgs field $\phi(x)$ $\equiv m(x)$, to which the relativistic Fermionic field $\Psi(x)$ couples through the Yukawa Lagrangian ${ }^{21,55,56} \mathcal{L}_{\mathrm{Y}}=-\phi \Psi^{\dagger} \beta \Psi \quad(\beta$ being a Pauli matrix). In the elementary particles Standard Model, ${ }^{57}$ such coupling is responsible for the masses of quarks and leptons. For $\phi(x) \equiv \phi_{0}$ (constant) $\mathcal{M} \nu_{\mathrm{F}}^{2}=\phi_{0}$, and the massive Fermion Dirac theory is recovered. 
We exploit the generalized Dirac physics governed by a total Lagrangian density $\mathcal{L}=\mathcal{L}_{\mathrm{f}}+\mathcal{L}_{\phi}$, where the Fermionic part is given by

$$
\mathcal{L}_{\mathrm{f}}=-\mathrm{i} \hbar \Psi^{\dagger} \frac{\partial}{\partial t} \Psi-\mathrm{i} \hbar v_{\mathrm{F}} \Psi^{\dagger} \alpha \frac{\partial}{\partial x} \Psi+\mathcal{L}_{\mathrm{Y}}
$$

and the scalar field part has the form

$$
\mathcal{L}_{\phi}=-\frac{1}{2}\left(\frac{\partial \phi}{\partial x}\right)^{2}-\frac{\xi}{4}\left(\phi^{2}-\phi_{0}^{2}\right)^{2}
$$

with the potential $V(\phi)$ (second term) assumed to have a double-well $\phi^{4}$ form; $\xi$ and $\phi_{0}$ are constants.

Henceforth, the Dirac equation (see the Methods Section) is generalized as

$$
E \Psi+\mathrm{i} \hbar v_{\mathrm{F}} \alpha \frac{\partial \Psi}{\partial x}-\beta \phi(x) \Psi=0
$$

In one dimension, the Fermion field is a two-component spinor $\Psi=\left(\psi_{\mathrm{u}}, \psi_{1}\right)^{T} ; \mathrm{u}$ and 1 stand, respectively, for the upper and lower components, and $\alpha$ and $\beta$ can be any two of the three Pauli matrices.

A graphene polygonal ring can be viewed as made of connected graphene nanoribbon fragments (here, we consider aGNRs). The excitations of an infinite aGNR are described by the $1 \mathrm{D}$ massive Dirac equation; see eq 22 with $\alpha=\sigma_{2}, \beta=\sigma_{1}$, and $\phi(x) \equiv \phi_{0}=\Delta / 2 \equiv\left|t_{1}-t_{2}\right|$. The two (in general) unequal hopping parameters $t_{1}$ and $t_{2}$ are associated with an effective 1D TB problem $)$ and are given ${ }^{58}$ by $t_{1}=-2 t \cos \left[p \pi /\left(\mathcal{N}^{W}+1\right)\right]$, $p=1,2, \ldots, \mathcal{N}^{W}$, and $t_{2}=-t ; \mathcal{N}^{W}$ is the number of carbon atoms specifying the width of the nanoribbon, and $t=2.7 \mathrm{eV}$ is the hopping parameter for $2 \mathrm{D}$ graphene. The effective ${ }^{58} \mathrm{~TB}$ Hamiltonian of an aGNR has a form similar to that used in trans-polyacetylene (a single chain of carbon atoms). In transpolyacetylene, the inequality of $t_{1}$ and $t_{2}$ (referred to as dimerization) is a consequence of the aforementioned Peierls distortion induced by the electron-phonon coupling. For an armchair graphene ring (AGR), this inequality is a topological effect associated with the geometry of the edge and the width of the ribbon. We recall that as a function of their width, $\mathcal{N}^{W}$, the aGNRs fall into three classes: (I) $\mathcal{N}^{W}=3 l$ (semiconducting, $\Delta$ $>0$ ), (II) $\mathcal{N}^{W}=3 l+1$ (semiconducting, $\Delta>0$ ), and (III) $\mathcal{N}^{W}=3 l+2$ (metallic $\left.\Delta=0\right), l=1,2,3, \ldots$

We adapt the "crystal" approach" ${ }^{52}$ to the $A B$ effect and introduce a virtual $\mathrm{DKP}^{51}$ relativistic superlattice (see the Methods Section). Charged Fermions in a perpendicular magnetic field circulating around the ring behave like electrons in a spatially periodic structure (period $\mathcal{D})$, with the magnetic flux $\Phi / \Phi_{0}\left(\Phi_{0}=h c / e\right)$ playing the role of the Bloch wave vector $k$, that is, $2 \pi \Phi / \Phi_{0}=k \mathcal{D}$ (see the cosine term in eq 10).

Naturally, nanorings with arms made of nanoribbon segments belonging to the semiconducting classes may be expected to exhibit a particle-hole gap (particle-antiparticle gap in relativistic quantum-field (RQF) theory). Indeed, this is found for a rhombic AGR (see the gap $\Delta$ in Figure 4a) with a width of $\mathcal{N}^{W}=12$ carbon atoms having type-I corners. Suprisingly, a rhombic armchair graphene nanoring of the same width $\mathcal{N}^{W}=12$, but having corners of type-II, demonstrates a different behavior, showing a "forbidden" band (with $\epsilon \approx 0$ ) in the middle of the gap region (see Figure 4b).
This behavior of rhombic AGRs with type-II corners can be explained through analogies with RQF theoretical models, describing single zero-energy Fermionic solitons with fractional charge $\mathrm{e}^{17,59}$ or their modifications when forming soliton/ antisoliton systems. ${ }^{17,60}$ (A solution of the equation of motion corresponding to eq 21 is a $Z_{2}$ kink soliton, $\phi_{\mathrm{k}}(x)$. The solution of eq 22 with $\phi=\phi_{\mathrm{k}}(x)$ is the Fermionic soliton.) We model the rhombic ring with the use of a continuous 1D Kronig-Penney ${ }^{51}$ model (see the Methods Section) based on the generalized Dirac eq 22, allowing variation of the scalar field $\phi(x)$ along the ring's arms. We find that the DKP model reproduces (see Figure $4 \mathrm{~d}$ ) the spectrum of the type-II rhombic ring (including the forbidden band) when considering alternating masses $\pm m_{0}$ associated with each half of the ring (see inset in Figure 4d).

In analogy with the physics of trans-polyacetylene (see remarks in the Introduction), the positive and negative masses correspond to two degenerate domains associated with the two possible dimerization patterns ${ }^{16,17} \cdots-t_{1}-t_{2}-t_{1}-t_{2}-\cdots$ and $\cdots-$ $t_{2}-t_{1}-t_{2}-t_{1}-\cdots$, which are possible in a single-atom chain. The transition zones between the two domains (here, two of the four corners of the rhombic ring) are referred to as the domain walls.

For a single soliton, a (precise) zero-energy Fermionic excitation emerges, localized at the domain wall. In the case of soliton-antisoliton pairs, paired energy levels with small positive and negative values appear within the gap. The TB spectrum in Figure $4 \mathrm{~b}$ exhibits a forbidden band of two paired $+/-$ levels, a property fully reproduced by the DKP model that employs two alternating mass domains (Figure 4d). The twofold forbidden band with $\epsilon \approx 0$ appears as a straight line due to the very small amplitude oscillations of its two members. $a_{0}=$ $0.246 \mathrm{~nm}$ is the graphene lattice constant, and $t=2.7 \mathrm{eV}$ is the hopping parameter.

The strong localization of a fraction of a Fermion at the domain walls (two of the rhombus' corners), characteristic of Fermionic solitons ${ }^{17}$ and of soliton/antisoliton pairs, ${ }^{60}$ is clearly seen in the TB density distributions (modulus of single-particle wave functions) displayed in Figure 5a. The TB $A$ (B) sublattice component of the TB wave functions localizes at the odd-numbered corners. These alternating localization patterns are faithfully reproduced (see Figure $5 b$ ) by the upper, $\psi_{w}$, and lower, $\psi_{1}$, spinor components of the continuum DKP model. The soliton-antisoliton pair in Figure $5 b$ generates an $e / 2$ charge fractionization at each of the odd-numbered corners, which is similar to the $e / 2$ fractionization familiar from polyacetylene.

The absence of a forbidden band (i.e., solitonic excitations within the gap) in the spectrum of the type-I rhombic nanorings (see Figure 4a) indicates that the corners in this case do not induce an alternation between the two equivalent dimerized domains (represented by $\pm m_{0}$ in the DKP model). Here, the corners do not act as topological domain walls. Nevertheless, direct correspondence between the TB and DKP spectra is achieved here too by using a variable Higgs field defined as $\phi(x)=m_{i}^{(n)}(x)$, with $m_{1}^{(n)}=m_{3}^{(n)}=0$ and $m_{2}^{(n)}=m_{0}=$ $0.15 t / \nu_{\mathrm{F}}^{2}$ (see the schematic inset in Figure $4 \mathrm{c}$ and the DKP spectrum plotted in the same figure).

\section{DISCUSSION}

In this paper, we focused on manifestations of relativistic and/ or nonrelativistic quantum behavior explored through theoretical considerations of transport in graphene nanostructures and 


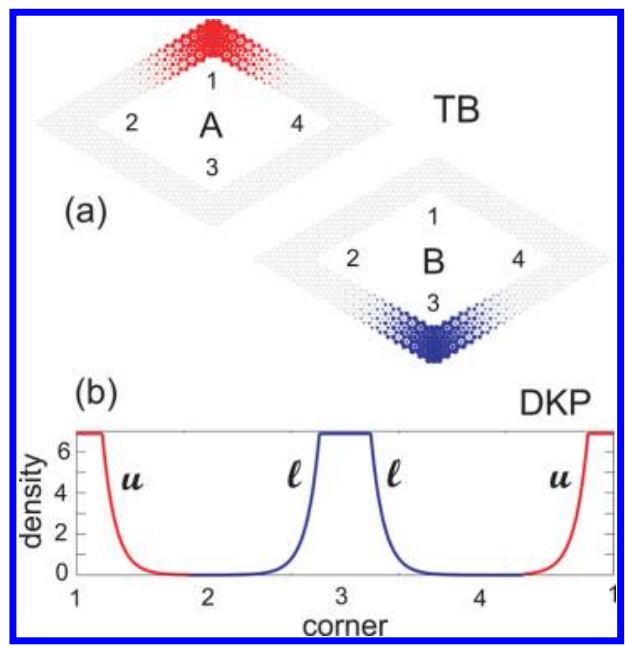

Figure 5. Wave functions for an excitation belonging to the "forbidden" solitonic band. (a) A sublattice (red) and $B$ sublattice (blue) components of the TB state with energy $\varepsilon=0.280 \times 10^{-4} t$ at $\Phi$ $=0$, belonging to the forbidden solitonic band of the type-II nanoring with $\mathcal{N}^{W}=12$ (see Figure 4b). (b) Upper (red) and lower (blue) spinor components for the corresponding state (forbidden band) according to the DKP spectrum (see Figure $4 \mathrm{~d}$ ), reproducing the TB behavior of the type-II nanoring with $\mathcal{N}^{W}=12\left(m_{0}=0.15 t / \nu_{\mathrm{F}}^{2}\right)$. The TB and DKP wave functions for all states of the solitonic band are similar to those displayed here. The wave functions here represent a pair of solitons. For contrast, see Figure 10 in ref 21, which portrays schematically the spinor $\Psi_{\mathrm{S}}$ for a single Fermionic soliton attached to a Higgs field with a smooth kink-soliton analytic shape $\phi_{\mathrm{k}}(x)=$ $\phi_{0} \tanh \left[(\xi / 2)^{1 / 2} \phi_{0} x\right] . \phi_{\mathrm{k}}(x)$ is a solution ${ }^{17,21}$ of the Lagrangian in eq 21. DKP densities are in units of $10^{-3} / a_{0}$.

spectral and topological effects in graphene nanorings in the presence of magnetic fields. In particular, we investigated the emergence of new behavior of electrons in atomically precise segmented GNRs with different edge terminations (armchair, zigzag, and mixed ones) and in graphene rings. To these aims, we have employed TB calculations of electronic states with and without applied magnetic fields, the nonequilibrium Green's function transport theory, and a newly developed Dirac continuum model that absorbs the valence-to-conductance energy gaps as position-dependent masses, including topological-in-origin mass barriers at the contacts between segments.

The electronic conductance has been found to exhibit FP oscillations, or resonant tunneling, associated with partial confinement and formation of a quantum box (resonant cavity) in the junction. Along with the familiar optical FP oscillations, exhibiting equal spacing between neighboring peaks, which we find for massless electrons in GNRs with metallic armchair central segments, we find other FP categories that differ from the optical one. In particular, our calculations reveal (a) a massive relativistic FP pattern exhibiting a valence-toconduction gap and unequal peak spacings; this pattern is associated with semiconducting armchair nanoribbon central segments, irrespective of whether the armchair leads are matallic or semiconducting; (b) a massive nonrelativistic FP pattern with $1 / L^{2}$ peak spacings but with a vanishing valenceto-conduction gap; this pattern is the one expected for the carriers in usual semiconductors described by the (nonrelativistic) Schrödinger equation, and it is associated with zigzag nanoribbon central segments, regardless of whether zigzag or metallic armchair leads are used.
Perfect quantized conductance flat steps were found only for uniform GNRs. In the absence of extraneous factors, like disorder, in our theoretical model, the deviations from the perfect quantized conductance steps were unexpected. However, this aforementioned behavior obtained through TBNEGF calculations is well accounted for by a 1D continuum Fermionic DFP interference theory (see the Methods Section). This approach employs an effective position-dependent mass term in the Dirac Hamiltonian to absorb the finite width (valence-to-conduction) gap in armchair nanoribbon segments, as well as the barriers at the interfaces between nanoribbon segments forming a junction. For zigzag nanoribbon segments, the mass term in the Dirac equation reflects the nonrelativistic Schrödinger-type behavior of the excitations. The carrier mass in zGNR segments is much larger than the particle mass in semiconducting aGNR segments. Furthermore, in the zGNR segments (which are always characterized by a vanishing valence-to-conduction energy gap), the mass corresponds simply to the carrier mass. In the aGNR segments, the carrier mass endows (in addition) the segment with a valence-toconduction energy gap, according to Einstein's relativistic energy relation (see eq 1 ).

We conclude with a brief discussion of the physics of electrons in segmented polygonal rings, which may be regarded as constructed by connecting GNR segments. Evaluation of the electronic states in a rhombic graphene nanoring under the influence of an applied magnetic field in the $A B$ regime and their analysis with the use of a relativistic quantum field theoretical model, unveils development of a topological-inorigin zero-energy soliton state and charge fractionization.

The above findings point to a most fundamental underlying physics, namely, that the topology of disruptions of the regular honeycomb lattice (e.g., variable width segments, corners, edges) generates a scalar potential field (position-dependent mass, identified ${ }^{21,22}$ also as a Higgs-type field), which when integrated into a generalized Dirac equation for the electrons provides a unifying framework for the analysis of transport processes through graphene segmented junctions and the nature of electronic states in graphene nanorings.

With growing activities and further improvements in the areas of bottom-up fabrication and manipulation of atomically precise $^{24-30}$ graphene nanostructures and the anticipated measurement of conductance through them, the above findings could serve as impetus and implements aiding the design and interpretation of future experiments.

\section{AUTHOR INFORMATION}

\section{Corresponding Author}

*E-mail: Uzi.Landman@physics.gatech.edu.

\section{Author Contributions}

I.R. and C.Y. performed the computations. C.Y., I.R. and U.L. analyzed the results. C.Y. and U.L. wrote the manuscript.

\section{Notes}

The authors declare no competing financial interest.

\section{ACKNOWLEDGMENTS}

This work was supported by a grant from the Office of Basic Energy Sciences of the U.S. Department of Energy under Contract No. FG05-86ER45234. Computations were made at the GATECH Center for Computational Materials Science. 


\section{REFERENCES}

(1) Nitzan, A.; Ratner, M. A. Electron Transport in Molecular Wire Junctions. Science 2003, 300, 1384-1389.

(2) Joachim, C.; Ratner, M. A. Molecular Electronics: Some Views on Transport Junctions and Beyond. Proc. Natl. Acad. Sci. U.S.A. 2005, 102, 8801-8808.

(3) Lindsay, S. M.; Ratner, M. A. Molecular Transport Junctions: Clearing Mists. Adv. Maters. 2007, 19, 23-31.

(4) Hod, O.; Rabani, E.; Baer, R. Magnetoresistance of Nanoscale Molecular Devices. Acc. Chem. Res. 2006, 39, 109-117.

(5) Bergfield, J. P.; Ratner, M. A. Forty Years of Molecular Electronics: Non-Equilibrium Heat and Charge Transport at the Nanoscale. Phys. Status Solidi B 2013, 250, 2249-2266.

(6) Rai, D.; Hod, O.; Nitzan, A. Magnetic Field Control of the Current through Molecular Ring Junctions. J. Phys. Chem. Lett. 2011, 2, 2118-2124.

(7) Kroto, H. W.; Heath, J. R.; O’Brien, S. C.; Curl, R. F.; Smalley, R.

E. C60: Buckminsterfullerine. Nature 1985, 318, 162-163.

(8) Iijima, S. Helical Microtubules of Graphitic Carbon. Nature 1991, $354,56-58$.

(9) Novoselov, K. S.; Geim, A. K.; Morozov, S. V.; Jiang, D.; Zhang, Y.; Dubonos, S. V.; Grigorieva, I. V.; Firsov, A. A. Electric Field Effect in Atomically Thin Carbon Films. Science 2004, 306, 666-669.

(10) Nakada, K.; Fujita, M.; Dresselhaus, G.; Dresselhaus, M. S. Edge State in Graphene Ribbons: Nanometer Size Effect and Edge Shape Dependence. Phys. Rev. B 1996, 54, 17954-17961.

(11) Son, Y. W.; Cohen, M. L.; Louie, S. G. Energy Gaps in Graphene Nanoribbons. Phys. Rev. Lett. 2006, 97, 216803.

(12) Wang, Z. F.; Li, Q.; Zheng, H.; Ren, H.; Su, H.; Shi, Q. W.; Chen, J. Tuning the Electronic Structure of Graphene Nanoribbons through Chemical Edge Modification: A Theoretical Study. Phys. Rev. B 2007, 75, 113406.

(13) Wakabayashi, K.; Sasaki, K.; Nakanishi, T.; Enoki, T. Electronic States of Graphene Nanoribbons and Analytical Solutions. Sci. Technol. Adv. Mater. 2010, 11, 054504 , and references therein.

(14) Castro Neto, A. H.; Guinea, F.; Peres, N. M. R.; Novoselov, K. S.; Geim, A. K. The Electronic Properties of Graphene. Rev. Mod. Phys. 2009, 81, 109-162.

(15) Katsnelson, M. I.; Novoselov, K. S.; Geim, A. K. Chiral Tunnelling and the Klein Paradox in Graphene. Nat. Phys. 2006, 2, $620-625$.

(16) Heeger, A. J.; Kivelson, S.; Schrieffer, J. R.; Su, W.-P. Solitons in Conducting Polymers. Rev. Mod. Phys. 1988, 60, 781-847.

(17) Jackiw, R.; Schrieffer, J. R. Solitons with Fermion Number 1/2 in Condensed Matter and Relativistic Field Theories. Nucl. Phys. B 1981, 190 [FS3], 253-265.

(18) Jackiw, R. Fractional and Majorana Fermions: The Physics of Zero-Energy Modes. Phys. Scr. 2012, 146, 014005.

(19) Peierls, R. F. Quantum Theory of Solids; Clarendon Press: Oxford, U.K., 1955.

(20) Romanovsky, I.; Yannouleas, C.; Landman, U. Patterns of the Aharonov-Bohm Oscillations in Graphene Nanorings. Phys. Rev. B 2012, 85, 165434

(21) Romanovsky, I.; Yannouleas, C.; Landman, U. Topological Effects and Particle Physics Analogies Beyond the Massless DiracWeyl Fermion in Graphene Nanorings. Phys. Rev. B 2013, 87, 165431.

(22) Yannouleas, C.; Romanovsky, I.; Landman, U. Beyond the Constant-Mass Dirac Physics: Solitons, Charge Fractionization, and the Emergence of Topological Insulators in Graphene Rings. Phys. Rev. B 2014, 89, 035432.

(23) Kivelson, S.; Chapman, O. I. Polyacene and A New Class of Quasi-One-Dimensional Conductors. Phys. Rev. B 1983, 28, 72367243.

(24) Cai, J. M.; Ruffieux, P.; Jaafar, R.; Bieri, M.; Braun, T.; Blankenburg, S.; Muoth, M.; Seitsonen, A. P.; Saleh, M.; Feng, X.-L.; et al. Atomically Precise Bottom-Up Fabrication of Graphene Nanoribbons. Nature 2010, 466, 470-473.

(25) Fuhrer, M. S. Graphene: Ribbons Piece-by-Piece. Nat. Mater. 2010, 9, 611-612.
(26) Huang, H.; Wei, D.; Sun, J.; Wong, S. L.; Feng, Y. P.; Castro Neto, A. H.; Wee, A. T. S. Spatially Resolved Electronic Structures of Atomically Precise Armchair Graphene Nanoribbons. Sci. Rep. 2012, 2, 983.

(27) van der Lit, J.; Boneschanscher, M. P.; Vanmaekelbergh, D.; Ijäs, M.; Uppstu, A.; Ervasti, M.; Harju, A.; Liljeroth, P.; Swart, I. Suppression of Electron-Vibron Coupling in Graphene Nanoribbons Contacted via A Single Atom. Nat. Commun. 2013, 4, 2023.

(28) Narita, A.; Feng, X.-L.; Hernandez, Y.; Jensen, S. A.; Bonn, M.; Yang, H. F.; Verzhbitskiy, I. A.; Casiraghi, C.; Hansen, M. R.; Koch, A. H. R. Synthesis of Structurally Well-Defined and Liquid-PhaseProcessable Graphene Nanoribbons. Nat. Chem. 2014, 6, 126-132.

(29) Hartley, C. S. Graphene Synthesis: Nanoribbons from the Bottom-Up. Nat. Chem. 2014, 6, 91-92.

(30) Vo, T. H.; Shekhirev, M.; Kunkel, D. A.; Morton, M. D.; Berglund, E.; Kong, L.; Wilson, P. M.; Dowben, P. A.; Enders, A.; Sinitskii, A. Large-Scale Solution Synthesis of Narrow Graphene Nanoribbons. Nat. Commun. 2014, 5, 3189.

(31) Blankenburg, S.; Cai, J. M.; Ruffieux, P.; Jaafar, R.; Passerone, D.; Feng, X.-L.; Müllen, K.; Fasel, R.; Pignedoli, C. A. Intraribbon Heterojunction Formation in Ultranarrow Graphene Nanoribbons. ACS Nano 2012, 6, 2020-2025.

(32) van Houten, H.; Beenakker, C. W. J.; Williamson, J. G.; Broekaart, M. E. I.; van Loosdrecht, P. H. M. Coherent Electron Focusing with Quantum Point Contacts in a Two-Dimensional Electron Gas. Phys. Rev. B 1989, 39, 8556-8575.

(33) Ji, Y.; Chung, Y. C.; Sprinzak, D.; Heiblum, M.; Mahalu, D.; Shtrikman, H. An Electronic Mach-Zehnder Interferometer. Nature 2003, 422, 415-418.

(34) vanWees, B. J.; van Houten, H.; Beenakker, C. W. J.; Williamson, J. G.; Kouwenhoven, L. P.; van der Marel, D.; Foxon, C. T. Quantized Conductance of Point Contacts in a TwoDimensional Electron Gas. Phys. Rev. Lett. 1988, 60, 848-850.

(35) Pascual, J. I.; Méndez, J.; Gómez-Herrero, J.; Baró, A. M.; Garcia, N.; Landman, U.; Luedtke, W. D.; Bogachek, E. N.; Cheng, H.P. Properties of Metallic Nanowires: From Conductance Quantization to Localization. Science 1995, 267, 1793-1795.

(36) Liang, W.; Bockrath, M.; Bozovic, D.; Hafner, J. H.; Tinkham, M.; Park, H. Fabry-Pérot Interference in a Nanotube Electron Waveguide. Nature 2001, 411, 665-669.

(37) Vaughan, J. M. The Fabry-Pérot Interferometer; Hilger: Bristol, U.K., 1989.

(38) Lipson, S. G.; Lipson, H.; Tannhauser, D. S. Optical Physics, 3rd ed.; Cambridge University Press: London, 1995; p 248.

(39) Rickhaus, P.; Maurand, R.; Liu, M.-H.; Weiss, M.; Richter, K.; Schönenberger, Ch. Ballistic Interferences in Suspended Graphene. Nat. Commun. 2013, 4, 2342.

(40) Datta, S. Quantum Transport: Atom to Transistor; Cambridge University Press: Cambridge, U.K., 2005.

(41) Lake, R.; Datta, S. Nonequilibrium Green's-Function Method Applied to Double-Barrier Resonant-Tunneling Diodes. Phys. Rev. B 1992, 45, 6670-6685.

(42) Ezawa, M. Peculiar Band Gap Structure of Graphene Nanoribbons. Phys. Status Solidi C 2007, 4, 489-492.

(43) Recher, P.; Trauzettel, B.; Rycerz, A.; Blanter, Ya. M.; Beenakker, C. W. J.; Morpurgo, A. F. Aharonov-Bohm Effect and Broken Valley Degeneracy in Graphene Rings. Phys. Rev. B 2007, 76, 235404.

(44) Bahamon, D. A.; Pereira, A. L. C.; Schulz, P. A. Inner and Outer Edge States in Graphene Rings: A Numerical Investigation. Phys. Rev. B 2009, 79, 125414.

(45) Wurm, J.; Wimmer, M.; Baranger, H. U.; Richter, K. Graphene Rings in Magnetic Fields: Aharonov-Bohm Effect and Valley Splitting. Semicond. Sci. Technol. 2010, 25, 034003.

(46) Hung Nguyen, V.; Niquet, Y. M.; Dollfus, P. Aharonov-Bohm Effect and Giant Magnetoresistance in Graphene Nanoribbon Rings. Phys. Rev. B 2013, 88, 035408.

(47) Hung Nguyen, V.; Niquet, Y. M.; Dollfus, P. The Interplay between the Aharonov-Bohm Interference and Parity Selective 
Tunnelingin Graphene Nanoribbon Rings. J. Phys.: Condens. Matter

2014, 26, 205301.

(48) Xu, H.; Heinzel, T.; Evaldsson, M.; Zozoulenko, I. V. Magnetic Barriers in Graphene Nanoribbons: Theoretical Study of Transport Properties. J. Phys.: Condens. Matter 2008, 77, 245401.

(49) Jiao, L.; Wang, X.; Diankov, G.; Wang, H.; Dai, H. Facile Synthesis of High-Quality Graphene Nanoribbons. Nat. Nanotechnol. 2010, 5, 321-325.

(50) Ritter, C.; Makler, S. S.; Latgé, A. Energy-Gap Modulations of Graphene Ribbons under External Fields: A Theoretical Study. J. Phys.: Condens. Matter 2008, 77, 195443.

(51) McKellar, B. H. J.; Stephenson, G. J. Relativistic Quarks in OneDimensional Periodic Structures. Phys. Rev. C 1987, 35, 2262-2271.

(52) Büttiker, M.; Imry, Y.; Landauer, R. Josephson Behavior in Small Normal One-Dimensional Rings. Phys. Lett. A 1983, 96, 365-367.

(53) Alberto, P.; Fiolhais, C.; Gil, V. M. S. Relativistic Particle in a Box. Eur. J. Phys. 1996, 17, 19-24.

(54) López-Villanueva, J. A.; Melchor, I.; Cartujo, P.; Carceller, J. E. Modified Schrödinger-Equation Including Nonparabolicity for the Study of a Two-Dimensional Electron Gas. Phys. Rev. B 1993, 48, 1626-1631.

(55) MacKenzie, R.; Palmer, W. F. Bags via Bosonization. Phys. Rev. D 1990, 42, 701-709.

(56) Bednyakov, V. A.; Giokaris, N. D.; Bednyakov, A. V. On Higgs Mass Generation Mechanism in the Standard Model. Phys. Part. Nucl. 2008, 39, 13-36.

(57) Griffith, D.; Introduction to Elementary Particles, 2nd ed.; WileyVCH: Weinheim, Germany, 2008.

(58) Zheng, H. X.; Wang, Z. F.; Luo, T.; Shi, Q. W.; Chen, J. Analytical Study of Electronic Structure in Armchair Graphene Nanoribbons. Phys. Rev. B 2007, 75, 165414.

(59) Jackiw, R.; Rebbi, C. Solitons with Fermion Number 1/2. Phys. Rev. D 1976, 13, 3398-3409.

(60) Jackiw, R.; Kerman, A. K.; Klebanov, I.; Semenoff, G. Fluctuations of Fractional Charge in Soliton Anti-Soliton Systems. Nucl. Phys. B 1983, 225, 233-246.

\section{NOTE ADDED AFTER ASAP PUBLICATION}

This paper was originally published on January 28, 2015. Due to a production error, there was an error in equation 10 . The corrected version was reposted on January 29, 2015. 